\title{
Site-specific colonic carcinoma in identical twins
}

\author{
STEPHEN WHITEHEAD \\ M.B., F.R.C.S.
}

Kent and Canterbury Hospital, Canterbury, Kent CT1 3NG

\begin{abstract}
Summary
The development of identically sited carcinomas of the colon in monozygotic twins is described. Presentation occurred within 2 years of each other. They are discussed in the context of the cancer-family syndrome.
\end{abstract}

\section{Introduction}

Carcinoma of the colon is common, and its presence in several members of a family may seem coincidental. In the absence of familial polyposis coli, if one member of a family is affected with colonic carcinoma, the other members have a 3 times greater than normal chance of developing the disease (Lovett, 1976).

Features which accentuate this risk include a high incidence of other adenocarcinomas in the family, multiple primaries and an early age of onset in the index case-the so-called cancer-family syndrome (Smith, 1976). In these families, the right half of the colon is affected in $65 \%$ of cases, whereas $65-85 \%$ of sporadic colonic carcinomas and those associated with familial polyposis coli occur on the left (Lynch, Lynch and Harris, 1977b).

These features suggest the existence of 2 different varieties of colonic carcinoma.

\section{Case histories}

A 32-year-old man (G.O.) presented with a year's history of upper abdominal pain, weight loss and melaena in October 1975. On investigation, his $\mathrm{Hb}$ was $6.7 \mathrm{gm} / \mathrm{dl}$ and a barium meal suggested duodenal ulceration.

He was given a blood transfusion and started on antacids.

After initial improvement, his symptoms recurred and a mass was palpable in the right hypochondrium. Barium enema showed a lesion at the hepatic flexure causing partial obstruction of the colon (Fig. 1a). At laparotomy 6 months later, a large ulcerating anaplastic carcinoma of the hepatic flexure was removed by right hemicolectomy. There were secondary deposits in the lymph nodes and the liver.

Over the next months he developed further abdominal pain, ascites and pleural effusions. Treatment was started with intermittent 5-day pulses of cytotoxic therapy, consisting of 5-fluorouracil, cyclophosphamide, methotrexate and vincristine. Despite this, he deteriorated and died 4 months after operation.

His identical twin (D.O.) developed similar symptoms in April 1977, but a barium enema showed no abnormality. Eleven months later he experienced further pain and on this occasion a mass was palpable. Repeat barium enema demonstrated a lesion of the transverse colon (Fig. 1b). At laparotomy, a mobile well differentiated adenocarcinoma of the right side of the transverse colon was removed by right hemicolectomy. Lymph node metastases were present in the mesentery.

He made an uneventful recovery, and subsequent abdominal lymphangiograms and an isotopic liver scan have shown no evidence of residual metastases. Pulsed cytotoxic therapy after an initial induction course has been started.

\section{Discussion}

The occurrence of a disease outside its normal pattern prompts investigation into the reason for its appearance. In the cases presented here, the brothers shared environmental as well as genetic factors which may be relevant to the production of their disease.

Lovett (1976) found 3 twin siblings among 209 cases of colonic carcinoma who had a family history of the disease, but none of the 3 non-index twins developed the disease.

Jarvik and Falek (1962) reviewed the development of carcinoma (in general) in 959 sets of twins and found a concordance rate of only $9.8 \%$ for monozygotic twins. This is less than might be expected, considering the accepted environmental and genetic evidence for the aetiology of malignant disease.

The incidence of carcinoma in twins under the age of $\mathbf{4 0}$ years is the same as in the single born (Osborne and De George, 1964).

There was no history of colonic carcinoma in their own or the preceding generation of the family 

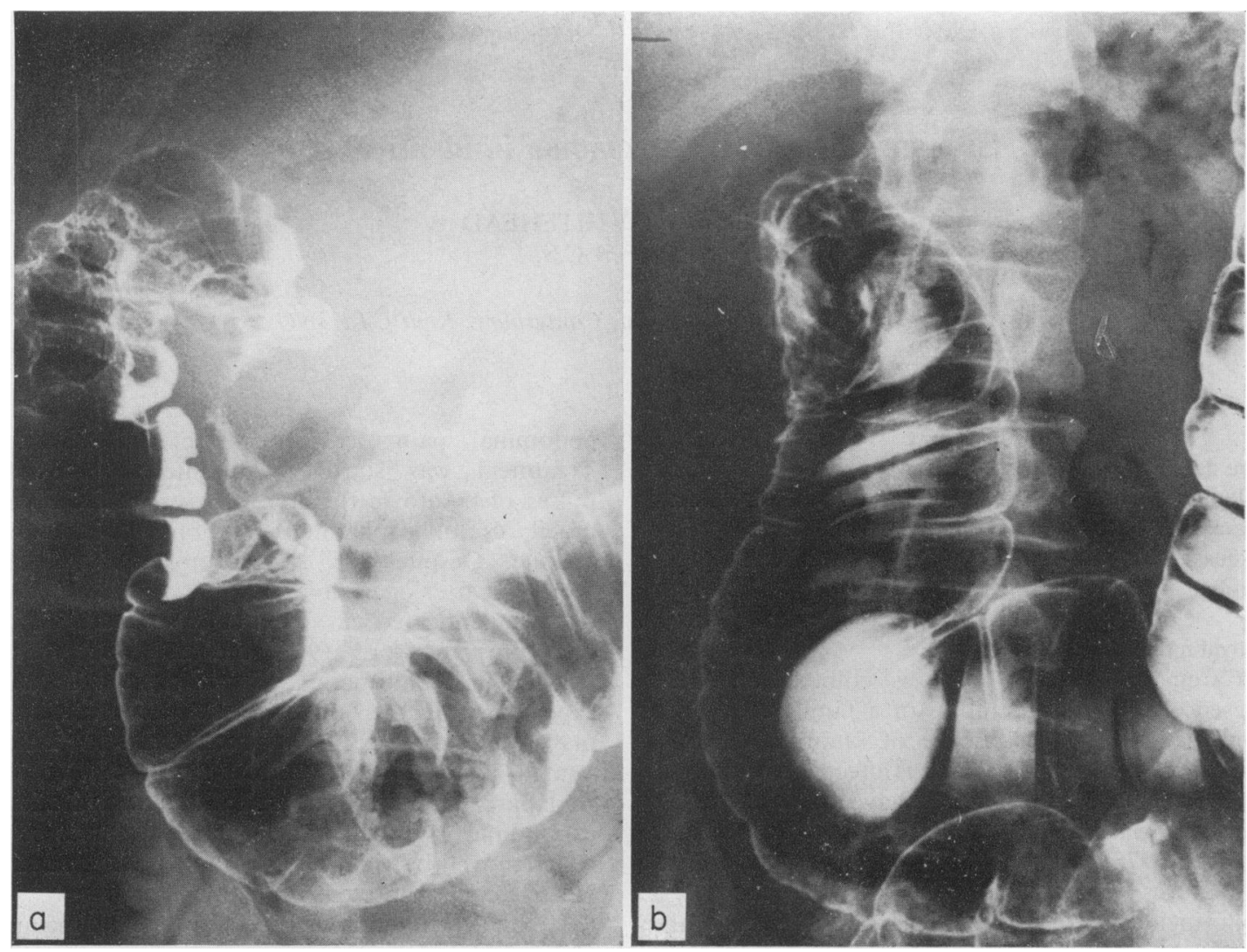

FIG. 1. Barium enema showing obstructive carcinoma of the transverse colon in (a) twin one; and (b) twin two.

(13 members). There is, however, strong evidence for a genetic aetiology of colonic carcinoma, although it is not proved beyond doubt. Dunstone and Knaggs (1972) found that in an affected family of 64 members, the incidence of colonic carcinoma came very close to that expected for transmission by a single dominant gene. In another report (Lynch et al., 1977a), a family of 79 members studied over 5 generations showed that the risk of developing colonic cancer among the offspring of affected parents was approximately $50 \%$, suggesting autosomal dominant inheritance.

So far as the author is aware, site-specific colonic carcinoma in monozygotic twins has not previously been recorded.

D.O. has 4 children, all of whom are well, but who will require careful screening as they grow up. Right-sided colonic growths are better detected by radiology than by endoscopy, so the risks of repeated barium enemas will have to be weighed against those of missing a developing growth. A reliable serological test would be of value, if one existed, and the patient and his children have been HLAtyped in case this may subsequently prove useful.

\section{Acknowledgments}

I thank Dr M.O. Rake and Mr M.R. Williams for their helpful criticism and for permission to report these cases.

\section{References}

Dunstone, G.H. \& KNaggs, T.W.L. (1972) Familial cancer of the colon and rectum. Journal of Medical Genetics, 9, 451 .

JARVIK, L.F. \& FALEK, A. (1962) Comparative data on cancer in ageing twins. Cancer, 15, 1009.

LOVETT, E. (1976) Family studies in carcinoma of the colon and rectum. British Journal of Surgery, 63, 13.

LYNCH, H.T., HARRIS, R.E., BARDAWIL, W.A., LYNCH, P.M., Guirgis, H.A., SWARTZ, M.J. \& LYNCH, J.F. (1977a) Management of hereditary site-specific colon cancer. Archives of Surgery, 112, 170.

LYNCH, P.M., LYNCH, H.T. \& HARRIS, R.E. (1977b) Heredit ary proximal colonic cancer. Diseases of the Colon and Rectum, 20, 661 .

Osborne, R.H. \& DE George, F.V. (1964) Neoplastic diseases in twins: evidence for pre- or perinatal factors conditioning cancer susceptibility. Cancer, 17, 1149.

Smith, W.G. (1976) The cancer family syndrome and familial clustering of solitary colorectal carcinoma. Diseases of the Colon and Rectum, 19, 126. 